UVX 2008 (2009) 163-167

(C) EDP Sciences, 2009

DOI: $10.1051 / \mathrm{uvx} / 2009026$

\title{
Production de rayons X par plasma ECR
}

\author{
N. Majeri ${ }^{1,2}$, C. Cachoncinlle ${ }^{1}$, S. Dozias ${ }^{1}$, E. Robert ${ }^{1}$, R. Viladrosa ${ }^{1}$, \\ J.-M. Pouvesle ${ }^{1}$, S. Milsant ${ }^{2}$ et M. Hugnot ${ }^{2}$ \\ ${ }^{1}$ GREMI, CNRS/Université d'Orléans, BP. 6744, 45067 Orléans, France \\ 2 INEL, Z.A. - C.D. 405, 45410 Artenay, France
}

\begin{abstract}
Résumé. Dans certaines conditions opératoires, les plasmas créés par résonance cyclotronique électronique, ECR, génèrent des rayons X sur une grande gamme d'énergie s'étendant de 1 à plus de $200 \mathrm{keV}$. Ce type de source à l'origine développée à l'école Polytechnique, est actuellement en cours d'étude au GREMI en collaboration avec l'industriel INEL qui exploite le brevet. La nouvelle source permet de produire des doses de $1 \mathrm{mGy} / \mathrm{s}$ à 1 mètre. On présentera les caractéristiques spectrales de la source en fonction de différents paramètres : pressions, puissances injectées, séparation des aimants. L'insertion d'une cible a permit d'obtenir une source plus intense et plus ponctuelle.
\end{abstract}

\section{INTRODUCTION}

La source ECR, résonance cyclotronique électronique, est une source plasma générée en chauffant les électrons par une onde électromagnétique, dans notre cas micro-onde. Nous avons étudié ici les performances de ce type de plasma comme source de rayonnement X continue. Une étude précédente a montré de possibles applications dans le domaine de la radiologie médicale [1]. Lorsque la fréquence cyclotronique de l'électron est la même que la fréquence de l'onde injectée, on a un transfert important de l'énergie de l'onde vers les électrons. Ce mécanisme est appelé "chauffage résonant". Les électrons sont confinés dans le plasma au moyen d'une bouteille magnétique. Lorsque qu'un électron voit un champ magnétique croissant, la conservation du moment magnétique induit un transfert de la vitesse parallèle vers la vitesse perpendiculaire de l'électron. Quand la vitesse parallèle devient nulle la particule retourne en arrière, d'où le piégeage de la particule par le gradient du champ magnétique. Le chauffage résonant associé au piégeage, permet aux électrons de passer plusieurs fois dans la zone de résonance et donc d'obtenir des électrons de grande énergie. Ces électrons chauds interagissent avec les autres particules du plasma ou avec les parois du réacteur émettant un rayonnement de Bremsstrahlung. Notre source fonctionne avec un générateur micro-onde de $2 \mathrm{~kW}$ à $2,45 \mathrm{Ghz}$, le champ magnétique nécessaire pour avoir la résonance $\left(\omega_{c}=q B / m\right)$ est donc de 0,0875 Tesla. La pression de travail est comprise entre de 1 à $20 \mathrm{mPa}$ et le spectre des photons $\mathrm{X}$ obtenu s'étend de 1 à $100 \mathrm{keV}$ et peut dans certaines conditions atteindre $200 \mathrm{keV}$. La dose maximum mesurée est de $1 \mathrm{mGy} / \mathrm{s}$ à 1 mètre du centre du réacteur. Le principal avantage de la source par rapport aux sources classiques de rayons $\mathrm{X}$, est la production de photons X sans utilisation de haute tension (classiquement entre 20 et $400 \mathrm{kV}$ ) et une grande flexibilité dans le choix des domaines d'énergies et des doses produites.

\section{DISPOSITIF EXPÉRIMENTAL}

Le réacteur plasma, voir figure 1, est une cavité de résonance cylindrique en aluminium de hauteur $10 \mathrm{~cm}$ et de diamètre $12.7 \mathrm{~cm}$, qui correspond à la longueur d'onde de la fréquence 2,45 Ghz. Deux aimants permanents, en terre rare, ayant une induction de surface de 0.5 tesla sont utilisés pour générer le champ magnétique. Le réacteur est équipé de cinq ouvertures : une pour l'injection des microondes, une pour l'injection des gaz ( $\mathrm{Ar}$ ou $\mathrm{AR}+\mathrm{O} 2)$, une pour la sortie des photons $\mathrm{X}$ et une pour le pompage. La gamme de pression de travail nécessaire à la production des $\mathrm{X}$ est de $10^{-3}$ à $4.10^{-2}$ pascal. 
La dernière sortie est utilisée pour l'insertion d'une cible (en tungstène), avec un système de déplacement 3D afin de positionner la cible pour optimiser la dose. Le tout est recouvert de plaques de plomb de $1 \mathrm{~cm}$ d'épaisseur.

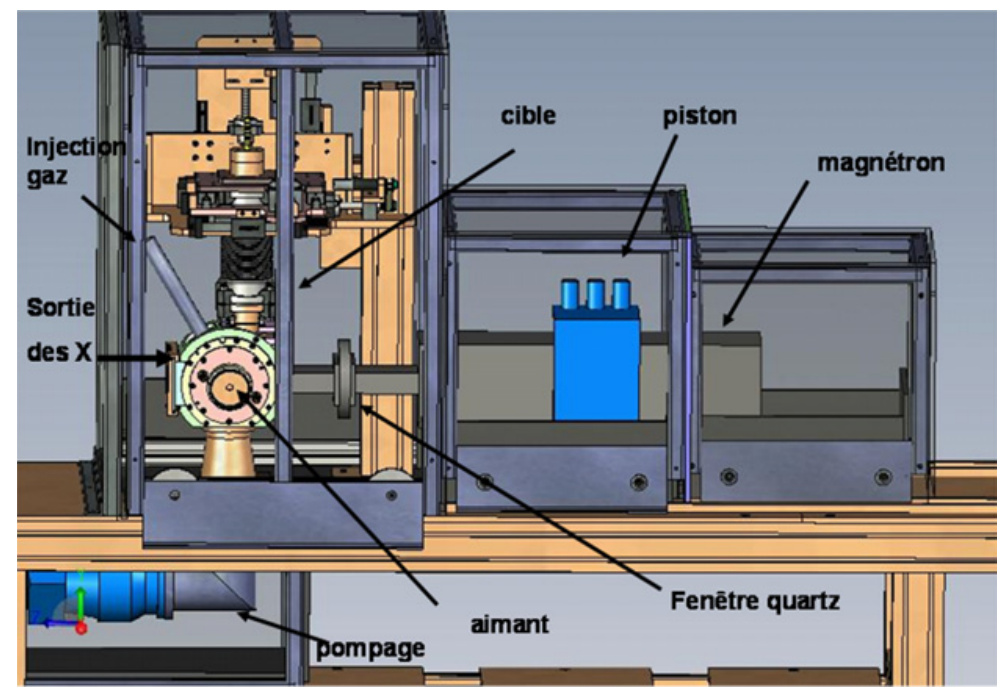

Figure 1. Schéma de la source.

Le système d'injection des micro-ondes est un système classique de chez Sairem, un magnétron qui génére l'onde de fréquence $2,45 \mathrm{GHz}$ d'une puissance max de $2 \mathrm{~kW}$, un adaptateur d'impédance à trois pistons et d'un circulateur qui dirige la puissance incidente vers la cavité et la puissance réfléchie vers une charge d'eau afin d'absorber la puissance pour ne pas endommager le générateur. Une fenêtre en quartz sépare le réacteur de l'adaptateur rempli d'air à la pression atmosphérique.

\section{STÉNOPÉ DES ANNEAUX ET DE LA CIBLE}

Nous avons réalisé des images du réacteur par sténopé, avec un diaphragme en plomb de 0,2 mm de diamètre et un film radiographique. La distance entre le film radiographique et le plomb est égale à celle entre le plomb et le centre du réacteur, pour avoir un grandissement de 1. Les anneaux observés sur les figures 2(a) et 2(b) dans deux conditions expérimentales différentes, de rayon 1 et $3 \mathrm{~cm}$ respectivement, résultent de l'émission $X$ produits par les électrons venant frapper les parois du réacteur. Le rayon de l'anneau correspond à la localisation de la zone de résonance (où le champs magnétique vaut $0,0875 \mathrm{~T}$ ), déterminé par la séparation (d) des aimants. L'introduction d'une cible de petite taille donne une source plus intense et plus ponctuelle. On peut avoir différentes tailles de source en fonction de la position de la cible et de la distance entre les deux aimants (voir figure 2(a) et figure 2(b)).

\section{4. ÉTUDE PARAMÉTRIQUE DU SPECTRE}

Une étude paramétrique du spectre, avec et sans cible a été effectué, pour différentes conditions opératoires de pression et de puissance micro-onde. Les spectres ont été mesurés au moyen d'un détecteur germanium de $25 \mathrm{~mm}$ de diamètre. Les spectres, en mode comptage de photons, ont été obtenus à une distance de 2 mètres. Sur les figures, l'énergie est exprimée en keV et l'axe des ordonnées représente le nombre de photons. On a aussi étudié l'influence de la séparation des aimants et des caractéristiques du plasma généré. 


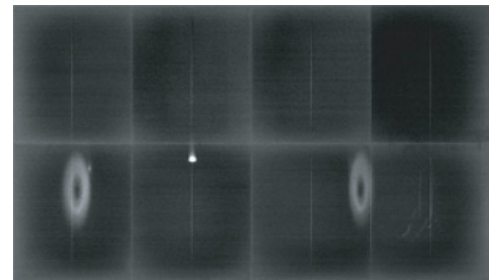

(a) Taille de cible de $1^{*} 1 \mathrm{~mm}^{2}$

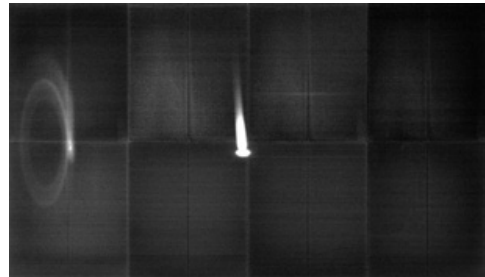

(b) Taille cible de $1^{*} 10 \mathrm{~mm}^{2}$

Figure 2. Image pinhole.

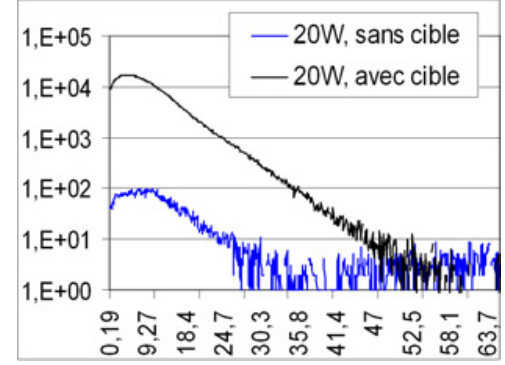

Figure 3. Spectre sans filtre.

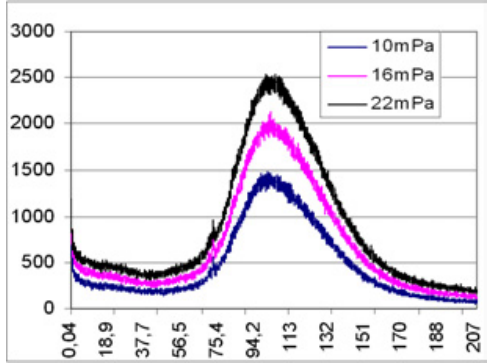

Figure 5. Influence de la pression. Spectre avec un filtre de $2 \mathrm{~cm}$ de fer (à $2 \mathrm{~kW}$ et $\mathrm{d}=120 \mathrm{~mm}$ ).

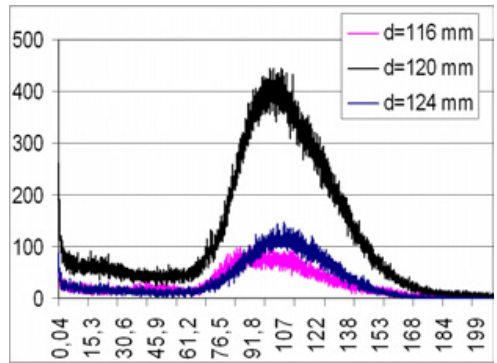

Figure 4. Influence de la distance entre les aimants sur l' aile du spectre à haute énegie avec un filtre de $2 \mathrm{~cm}$ de fer (à $2 \mathrm{~kW}$ et $7 \mathrm{mPa}$ ).

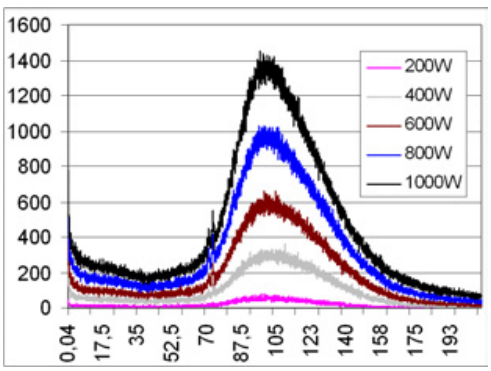

Figure 6. Influence de la puissance micro ondes. Spectre avec un filtre de $2 \mathrm{~cm}$ de fer (à $10 \mathrm{mPa}$ et $\mathrm{d}=120 \mathrm{~mm}$ ).

\subsection{Influence de la cible}

La figure 3 donne les spectres pour une puissance injectée de $20 \mathrm{~W}$ sans cible et avec cible. On observe un facteur 100 entre le spectre avec cible à $20 \mathrm{~W}$ et le spectre sans cible. L'ajout d'une cible permet donc non seulement d'avoir une cible plus ponctuelle mais aussi une dose beaucoup plus intense. 


\subsection{Influence de la séparation des aimants}

Le spectre présenté figure 3 montre l'influence de la séparation des aimants sur l'aile hautes énergies du spectre, où l'on a utilisé un filtre de $2 \mathrm{~cm}$ en fer. La séparation optimale pour avoir des photons de plus hautes énergies correspond au cas où la résonance (où le champ magnétique vaut $0,0875 \mathrm{~T}$ ) se trouve sur l'axe de symétrie entre les deux aimants.

\subsection{Influence de la pression et de la puissance}

La production de photon croit avec la pression, figure 5, jusqu'à une limite fonction de la puissance magnétron injectée. A pression trop élevée les collisions entraînent une thermalisation des électrons chauds rendant la production de rayonnement $\mathrm{X}$ inopérante. Pour continuer à augmenter le monbre de photon $\mathrm{X}$ produits il faut augmenter la puissance micro-onde comme pour la pression. La dose est proportionnelle a la puissance micro-ondes injectée (voir figure 5). En faite pour une pression donnée, il existe une puissance micro-onde limite au-delà de laquelle la production des X n'augmente plus, jusqu'à l'apparition d'instabilité qui dégrade la production de rayonnement. Par example pour une pression de $3 \mathrm{mPa}$ la puissance micro-ondes injectée limite est de $750 \mathrm{~W}$.

\subsection{Influence de l'ajout d'oxygène}

Il est connu dans les sources d'ions ECR que l'ajout d'une petite portion d'un gaz plus léger que le gaz principal augmente le nombre d'électrons énergétiques. Cet effet est également observé ici (figure 7). Pour l'expliquer plusieurs hypothèses ont été avancées, comme le transfert de l'énergie des ions les plus lourds vers les ions plus légers par collision [2], l'augmentation du temps de confinement due à la diminution de la charge moyenne, qui diminue les pertes des électrons [3], ou encore une baisse du potentiel plasma qui donne un meilleur confinement des ions et entraîne une meilleur stabilité du plasma [4]. Mais aucune des explications ne sont encore satisfaisantes car un effet de la masse a été aussi reporté [5], où 3 isotopes de l'oxygène ont été testés, le plus lourd ayant donné les meilleurs résultats. Des études sont en cours pour tenter d'identifier le processus dominant.

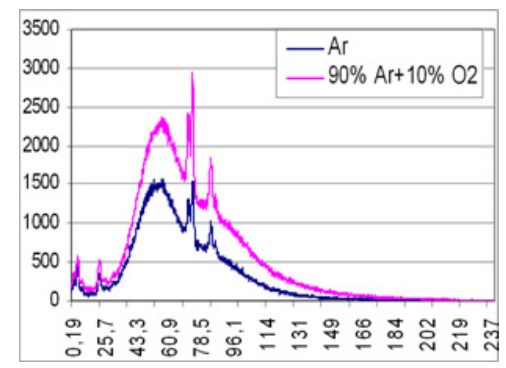

Figure 7. Influence de l'ajout d'oxygène. Spectre avec un filtre de $16 \mathrm{~cm}$ d'aluminium (à $2 \mathrm{~kW}, 20 \mathrm{mPa}$ et $\mathrm{d}=120 \mathrm{~mm}$ ).

\section{CONCLUSION}

Une nouvelle source de rayonnement $\mathrm{X}$, donnant des $\mathrm{X}$ d'énergie de 1 à $100 \mathrm{keV}$ et une dose maximum mesurée de $1 \mathrm{mGy} / \mathrm{s}$ à 1 mètre, avec une pression de travail de 1 à $20 \mathrm{mPa}$, a été développée et étudiée au GREMI. L'introduction d'une cible en tungstène a permit d'avoir une source plus intense et plus ponctuelle de $1 * 1$ à $1 * 10 \mathrm{~mm}^{2}$ suivant la taille de la cible. Cette source, très stable, peut fonctionner des heures dans des conditions de décharges établies. Des applications dans le domaine de la cristallographie et du contrôle industriel sont envisagées. 


\section{Références}

[1] Gaudin C., Lamoureux M., Rouillé C., Physics in Medicine and Biology 46, (2001) 835-851.

[2] T.A. Antaya, J. Phys. (Paris) Cl 50 (1989) 707.

[3] R. Geller, Ann. Rev. Nucl. Part. Sci. 40 (1990) 15. R. Geller et al., Proceedings of the 8th International Workshop on ECR Ion Sources, East Lansing, (1987), p. 1.

[4] Z. Q. Xie and C. M. Lyneis, Review of Scientific Instruments, 65, No. 9, (1994), p. 2947. O. Tarvainen, P. Suominen, T. Ropponen, T. Kalvas, P. Heikkinen and H. Koivisto, Rev. Sci. Instrum., 76, (9), (2005).

[5] A.G. Drentje and J. Sijbring, Pro. ECRIS 10. Report No ORNL CONF-9011136. 17 (1990); A.G. Drentje, 6th ICIS Sept. 10-16, Vancouver (1995). 